\title{
Details of the drag curve near the onset of vortex shedding
}

Ronald D. Henderson

Citation: Physics of Fluids 7, 2102 (1995); doi: 10.1063/1.868459

View online: http://dx.doi.org/10.1063/1.868459

View Table of Contents: http://aip.scitation.org/toc/phf/7/9

Published by the American Institute of Physics

\section{Articles you may be interested in}

On the drag of two-dimensional flow about a circular cylinder

Physics of Fluids 16, 3828 (2004); 10.1063/1.1789071

Defining a universal and continuous Strouhal-Reynolds number relationship for the laminar vortex shedding of a circular cylinder

The Physics of Fluids 31, 2742 (1998); 10.1063/1.866978

Laminar flow past a rotating circular cylinder

Physics of Fluids 11, 3312 (1999); 10.1063/1.870190

Onset of laminar separation and vortex shedding in flow past unconfined elliptic cylinders

Physics of Fluids 26, 023601 (2014); 10.1063/1.4866454

Simulation of three-dimensional flow around a square cylinder at moderate Reynolds numbers

Physics of Fluids 11, 288 (1999); 10.1063/1.869879

On the relationship of effective Reynolds number and Strouhal number for the laminar vortex shedding of a heated circular cylinder

Physics of Fluids 12, 1401 (2000); 10.1063/1.870391

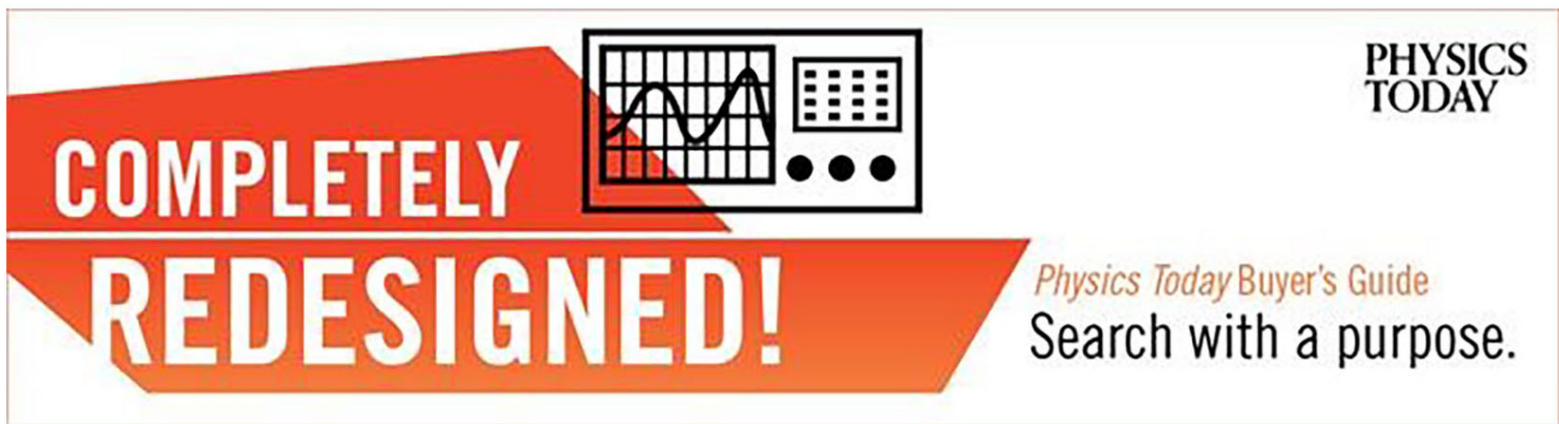




\title{
Details of the drag curve near the onset of vortex shedding
}

\author{
Ronald D. Henderson \\ Aeronautics and Applied Mathematics, California Institute of Technology, Pasadena, California 91125
}

(Received 21 February 1995; accepted 1 May 1995)

A closer look at the drag curve for a circular cylinder near the onset of vortex shedding reveals that there is a sharp transition in the forces acting on a body moving through a fluid when it produces an unsteady wake. In this Letter results from high-resolution computer simulations are presented to quantify the change in viscous drag, pressure drag, and base pressure coefficients. (c) 1995 American Institute of Physics.

Most textbooks dealing with the subject of fluid mechanics include the case of "flow past a circular cylinder" as the classic example of a bluff body. But much of the intricate detail and interesting behavior of this highly complex flow is often hidden under a relatively smooth drag vs. Re curve whose only outstanding feature seems to be the "drag crisis" at $R e \approx 5 \times 10^{5}$. In this Letter we look at an interesting feature at the other end of the drag curve, where $R e \approx 50$ : the response of cylinder drag to the onset of unsteadiness and vortex shedding.

The specific case we are considering here is a circular cylinder of diameter $d$ immersed in a fluid with density $\rho$ and dynamic viscosity $\mu$, moving at a free-stream velocity of $U_{\infty}$. These are the only parameters for an incompressible flow and the combination $R e \equiv \rho U_{\infty} d / \mu$ defines the Reynolds number. When $R e$ is less than 50 the flow is steady and symmetric about the centerline of the wake. Even at small values of the Reynolds number, say $R e \approx 10$, the flow separates from the surface of the cylinder and forms a pair of bound vortices in the near wake. At $R e \approx 50$ this configuration becomes unstable and the process of vortex shedding begins, resulting in the well-known Kármán vortex street. This qualitative description of low-speed flow past a circular cylinder is well known and much studied, but the exact details of the drag curve in this range are not. ${ }^{1}$

Contributions to the drag force on a cylinder can be measured in at least three ways: the viscous drag coefficient, the pressure drag coefficient, and the base suction coefficient. The first two are the components of the total drag and the third is simply another way to "characterize" the drag that is more sensitive to the dynamics of the wake (and easier to measure). Experimental values for these quantities are difficult to obtain, especially at $R e \leqslant 10^{3}$, and the available data show considerable scatter, so much so that it is impossible to draw a precise trend from the experiments alone. The results presented here are from high-resolution computer simulations of flow past an infinitely long circular cylinder-the mathematically ideal case. Near the onset of vortex shedding the wake exhibits a slow, time-periodic "waviness" and intuitively we might expect that the drag changes smoothly as the Reynolds number increases. We will see that it does so sharply, that the slope of the $C_{D}-R e$ curve changes discontinuously as the flow becomes unsteady. The remainder of this Letter quantifies how each contribution to the drag varies near the onset of vortex shedding.

The flowfield is obtained by solving the twodimensional, time-dependent Navier-Stokes equations. These equations are solved approximately using a spectral element method with resolution parameters (202 elements, 8th order polynomial basis) that were determined from a convergence study at $R e=190$, well above the range we are interested in. The algebraic systems for the velocity and pressure were solved directly using 64-bit arithmetic. Although the spectral element method has the advantage of low numerical dissipation and dispersion errors, any accurate simulation should produce identical results. A full description of the method is beyond the scope of this Letter, but the details may be found in Ref. 2. This approach has the conceptual advantage that the velocity and pressure fields are continuous, so the corresponding forces can be computed by direct integration of (some quantity) over the surface of the cylinder. The viscous and pressure forces are given by:

$F_{f}=-\oint \mu\left(\nabla \mathbf{u}+(\nabla \mathbf{u})^{T}\right) n_{x} d s, \quad F_{p}=\oint p n_{x} d s$,

and the corresponding force coefficients are normalized by the dynamic pressure, $q \equiv \frac{1}{2} \rho U_{\infty}^{2}$, acting on a unit span $l$ of the cylinder:

$$
C_{D f}=F_{f} / q d l, \quad C_{D_{P}}=F_{p} / q d l .
$$

The base pressure coefficient is a point measurement given by $C_{p b}=\left(p_{b}-p_{\infty}\right) / q$, where $p_{b}$ is the time-averaged pressure at the base ( 180 degrees from the front) of the cylinder.

Measurements for the steady flow are taken in the following way. The first simulation is started from an initial condition of uniform velocity at $R e=25$ and stopped when it reaches a steady state. Then the drag and base pressure coefficients are computed from the surface distribution of shear stress and pressure. This solution gives the initial condition for the next case, $R e=30$. We continue in increments of $\Delta R e=5$ until the time-dependent calculation no longer converges to a steady-state solution, taking the loose definition of "steady-state" to mean the solution changes by less than a factor of $10^{-4}$ in 50 time units. This part of the curve is shown using solid symbols in the figures, and ends at $R e=60$. 


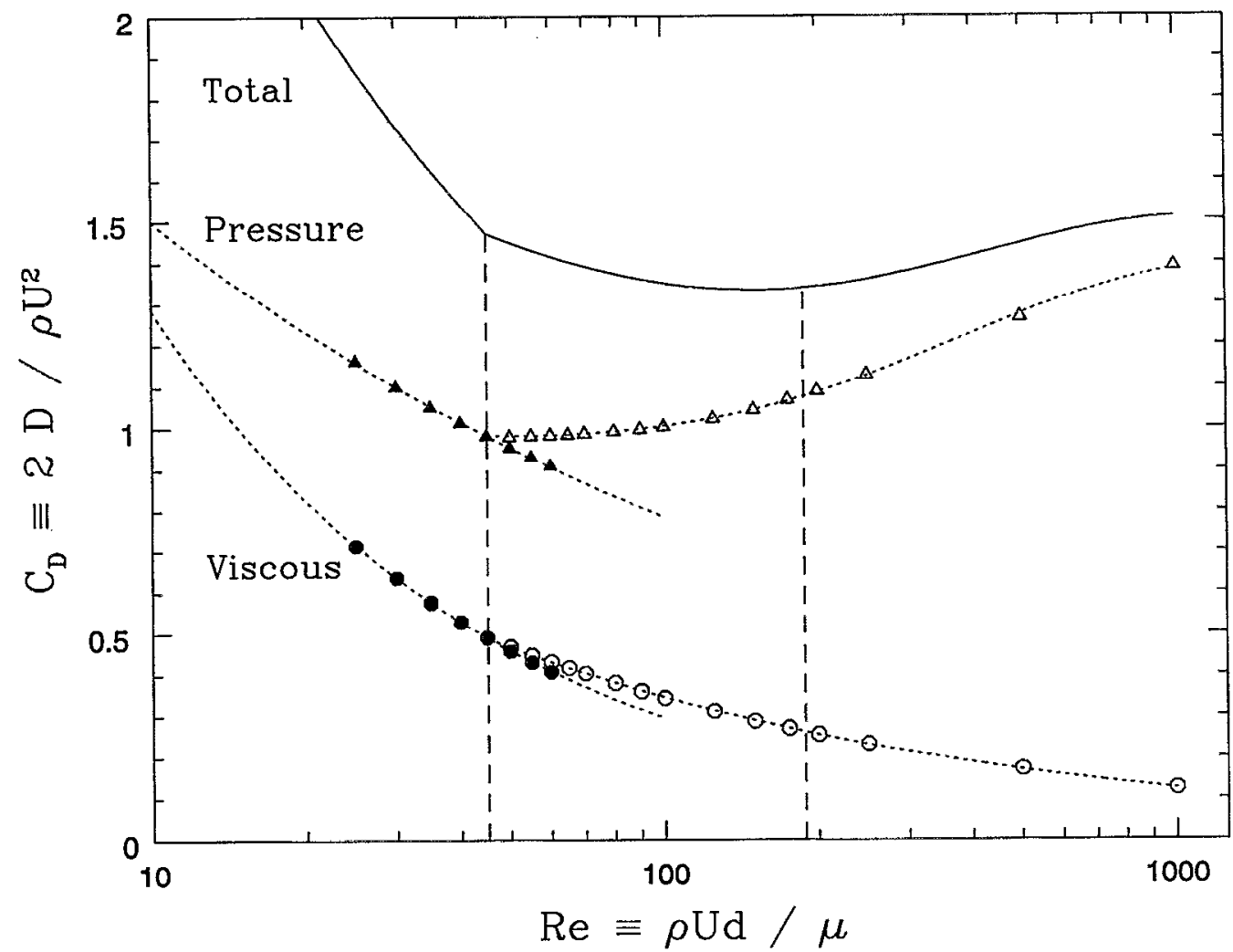

FIG. 1. Pressure and viscous drag coefficients versus Reynolds number for two-dimensional flow past a circular cylinder: $(\boldsymbol{\Theta}, \mathbf{\Delta})=$ steady wake, $(O, \Delta)=$ vortex shedding; the vertical dashed lines are explained in the following figure.

Above this Reynolds number, perturbations, even at the "infinitesimal" level of roundoff error, grow too quickly to measure any steady drag force, and the calculation converges to the time-periodic solution corresponding to laminar vortex shedding. Now we reduce the Reynolds number in the same amount until we cross back onto the drag curve for the steady wake, that is until the time-dependent calculation once again stabilizes, returning to a steady-state solution. The cross-over falls between $R e=45$ and 50, and agrees nicely with the critical value $R e_{1}=46 \pm 1$ obtained both in experimental observations and numerical stability calculations. ${ }^{3,4}$ Simulations at higher Reynolds numbers are simply continued from this curve. For these cases all quantities are averaged over one shedding cycle, and mean values are shown as open symbols in the figures. Note that the computational algorithm is identical for all cases and that with careful control of numerical dissipation no finite-amplitude disturbance is required to trigger vortex shedding.

Computed drag forces on a circular cylinder as a function of Reynolds number are shown in Fig. 1. Values corresponding to a steady wake are shown as solid symbols, and mean values corresponding to periodic vortex shedding are shown as open symbols. The first dashed line marks the critical Reynolds number $R e_{1}$ (defined above) where the wake becomes temporally unstable and vortex shedding begins. For $R e>R e_{1}$ the drag makes a sharp transition to the second family of curves; $C_{D f}$ continues to decrease (as it does for all values of $R e$ ) but $C_{D P}$ immediately increases.

The distinct change from a steady to unsteady wake is also shown clearly by the plot of base pressure in Fig. 2 . This figure includes careful experimental measurements by Williamson and Roshko ${ }^{5}$ that support the simulations and illustrate the importance of the second dashed line: it marks the next critical Reynolds number, $R e_{2}=188.5 \pm 1$, where the wake becomes (absolutely) linearly unstable to threedimensional disturbances. ${ }^{6}$ In contrast to the onset of vortex shedding, the onset of three-dimensionality appears to be a soft transition with a small range of hysteresis. It is interesting that the secondary instability occurs near the point where the total drag curve would begin to rise, so that the values of $C_{D}$ observed in experiments continually decrease up to $R e \approx 1000$.

It is uscful to look at the underlying form of the drag curves. Measurements from the simulations are accurately reproduced by the following simple functions. First is a two parameter fit (power law) for skin friction and steady pressure forces:

$$
f(x)=a_{0} / x^{a_{1}}
$$

In the unsteady case, time-averaged pressure forces can be represented by the four parameter fit:

$$
f(x)=a_{0}-a_{1} x^{a_{2}} \exp \left(a_{3} x\right) .
$$

The coefficients and corresponding chi-squared error, listed in Table I, were computed using a maximum likelihood estimator. Keep in mind these are simply fits to the measured data, and are not derived from or necessarily related to the Navier-Stokes equations. However, they do suggest limiting 


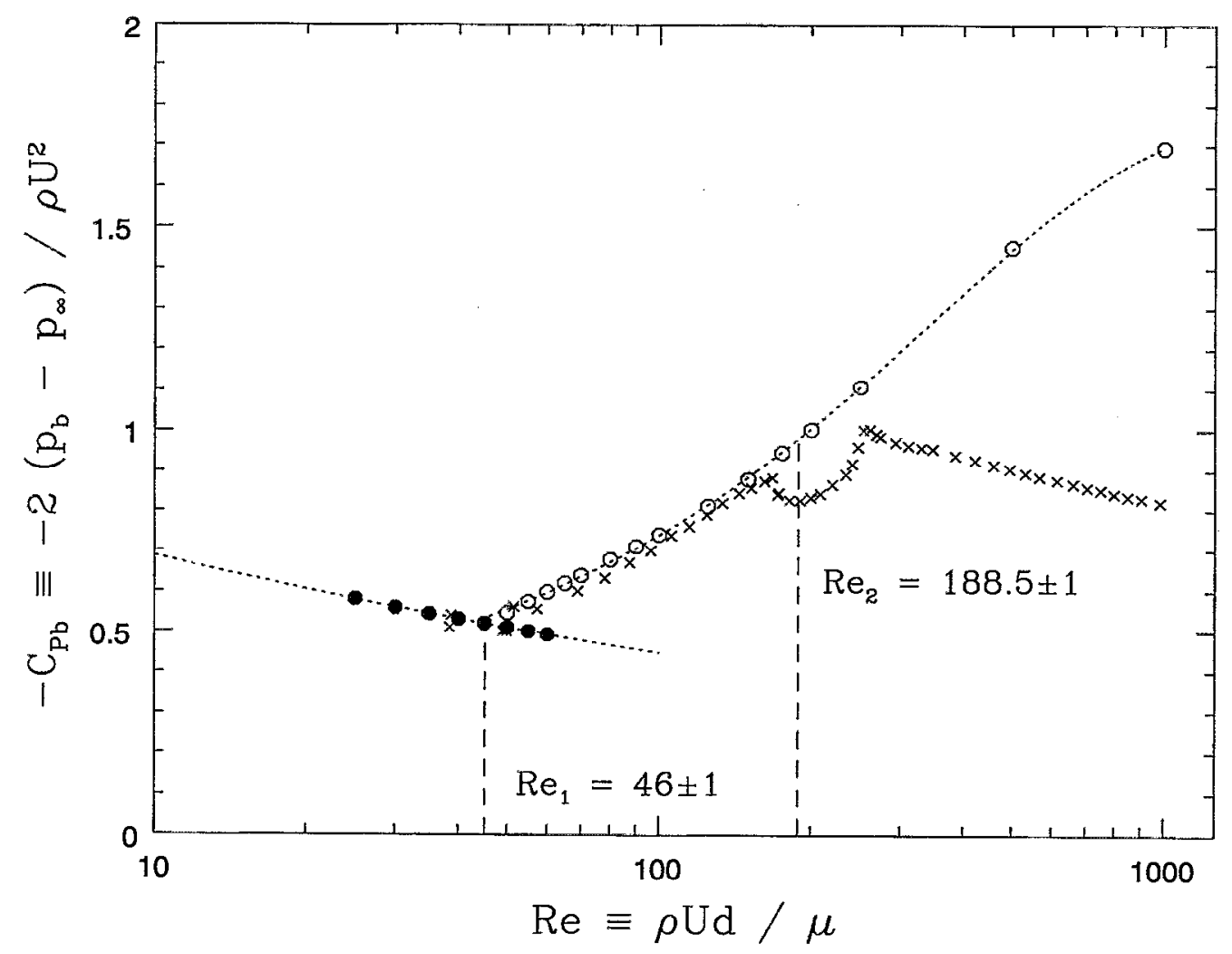

FIG. 2. Base pressure coefficients: $\theta=$ steady wake, $O=$ vortex shedding; $\times=$ experimental measurements. Vertical dashed lines mark the critical Reynolds number for (1) temporal instability and (2) three-dimensional instability.

values for the force coefficients corresponding to 2D flow past a cylinder as $R e \rightarrow \infty$, a regime still open to much speculation. Even the best calculations to date for the steady laminar flow have only reached $R e=600$, and the implications for higher Reynolds numbers are somewhat inconclusive. ${ }^{7}$ In theoretical fluid mechanics, these are still important and unanswered questions!

TABLE I. Functional forms for each of the drag curves: (upper) steady wake, (lower) vortex shedding.

\begin{tabular}{lccccc}
\hline \hline Drag curve & $a_{0}$ & $a_{1}$ & $a_{2}$ & $a_{3}$ & $\chi^{2}$ error \\
\hline$C_{D f}$ & 5.6106 & 0.6400 & $\cdot$ & $\cdot$ & $9.8 \times 10^{-7}$ \\
$C_{D p}$ & 2.8676 & 0.2815 & $\cdot$ & $\cdot$ & $1.1 \times 10^{-5}$ \\
$-C_{p b}$ & 1.0521 & 0.1854 & $\cdot$ & $\cdot$ & $3.8 \times 10^{-6}$ \\
\hline$C_{D f}$ & 2.5818 & 0.4369 & $\cdot$ & $\cdot$ & $4.7 \times 10^{-6}$ \\
$C_{D p}$ & 1.4114 & 0.2668 & 0.1648 & $-3.375 \times 10^{-3}$ & $3.7 \times 10^{-5}$ \\
$-C_{p b}$ & 1.7826 & 1.6575 & -0.0427 & $-2.660 \times 10^{-3}$ & $2.5 \times 10^{-4}$ \\
\hline \hline
\end{tabular}

\section{ACKNOWLEDGMENTS}

This question about the drag force grew out of discussions within the Bluff Body Wakes Group at GALCIT, and in particular with Anatol Roshko. The computations were performed at Caltech with the support of the National Science Foundation under Grant No. CDA-9318145, and the Office of Naval Research under Grant No. N00014-94-1-0793.

\footnotetext{
'A. Roshko, "Perspectives on bluff body aerodynamics," J. Wind Eng. 49, 79 (1993).

${ }^{2}$ R. D. Henderson, "Unstructured spectral element methods: Parallel algorithms and simulations." Ph.D. thesis, Princeton University, 1994.

${ }^{3}$ M. Provensal, C. Mathis, and L. Boyer, "Bénard-von Kármán instability: Transient and forced regimes," J. Fluid Mech. 182, 1 (1987).

${ }^{4}$ C. P. Jackson, "A finite-element study of the onset of vortex shedding in flow past variously shaped bodies," J. Fluid Mech. 182, 23 (1987).

${ }^{5} \mathrm{C}$. H. K. Williamson and A. Roshko, "Measurements of base pressure in the wake of a cylinder at low Reynolds numbers," Z. Flugwiss. Weitraumforsch. 14, 38 (1990).

${ }^{6}$ D. Barkley and R. D. Henderson, "Three-dimensional Floquet stability analysis of the wake of a circular cylinder," submitted to J. Fluid Mech. (1995).

${ }^{7} \mathrm{~B}$. Fornberg, "Steady viscous flow past a circular cylinder up to Reynolds number 600," J. Comput. Phys. 61, 297 (1985).
} 